\title{
Probing the Equation of State of Nuclear Matter via Neutron Star Asteroseismology
}

\author{
Hajime Sotani,, , * Ken'ichiro Nakazato, ${ }^{2}$ Kei Iida $^{3}$ and Kazuhiro Oyamatsu ${ }^{4}$ \\ ${ }^{1}$ National Astronomical Observatory of Japan, 2-21-1 Osawa, Mitaka, Tokyo 181-8588, Japan \\ ${ }^{2}$ Faculty of Science 85 Technology, Tokyo University of Science, 2641 Yamazaki, Noda, Chiba 278-8510, Japan \\ ${ }^{3}$ Department of Natural Science, Kochi University, 2-5-1 Akebono-cho, Kochi 780-8520, Japan \\ ${ }^{4}$ Department of Human Informatics, Aichi Shukutoku University, 9 Katahira, Nagakute, Aichi 480-1197, Japan
}

(Dated: August 7, 2018)

\begin{abstract}
We general relativistically calculate the frequency of fundamental torsional oscillations of neutron star crusts, where we focus on the crystalline properties obtained from macroscopic nuclear models in a way depending on the equation of state of nuclear matter. We find that the calculated frequency is sensitive to the density dependence of the symmetry energy, but almost independent of the incompressibility of symmetric nuclear matter. By identifying the lowest-frequency quasi-periodic oscillation in giant flares observed from soft gamma-ray repeaters as the fundamental torsional mode and allowing for the dependence of the calculated frequency on stellar models, we provide a lower limit of the density derivative of the symmetry energy as $L \simeq 50 \mathrm{MeV}$.
\end{abstract}

PACS numbers: 04.40.Dg, 21.65.Ef, 26.60.Gj, 97.10.Sj

Observations of the global oscillations of stars play a significant role in probing the properties of stellar matter, as in the case of the Sun. Studies in this direction are often referred to as asteroseismology. Oscillations of neutron stars are expected to give an insight to the properties of matter under conditions of ultra-high density. Recent observations of the quasi-periodic oscillations (QPOs) in giant flares from soft gamma-ray repeaters (SGRs) [1] suggest that a long-awaited evidence for the neutron star oscillations has been given with the help of coupling of gigantic magnetic fields with the solid crust. So far, three giant flares have been detected from SGR 0526-66, SGR 1900+14, and SGR 1806-20, and, through the timing analysis of the X-ray afterglow, the QPOs with frequencies in the range from tens $\mathrm{Hz}$ up to a few $\mathrm{kHz}$ have been discovered [1]. Many theoretical attempts to explain the observed QPO frequencies have been done in terms of the torsional oscillations in the crustal region and/or the magnetic oscillations (e.g., Refs. 2-9]). If the QPOs in giant flares are associated with the crustal oscillations, the properties of inhomogeneous nuclear matter in the crust could be clarified [10 12 .

The outer part of neutron stars can be described as follows. Below an ionic ocean in the vicinity of the surface, a bcc Coulomb lattice of nuclei embedded in a roughly uniform electron gas is considered to compose a crustal region. In this region, nuclei become gradually neutronrich with increasing density and even drip neutrons at a density of about $4 \times 10^{11} \mathrm{~g} \mathrm{~cm}^{-3}$. Near normal nuclear density, this crustal region is considered to melt into uniform nuclear matter. Just before melting, roughly spherical nuclei are so closely packed that global deformations into rodlike nuclei could occur. As the density increases further, possible changes of the nuclear shape are rod, slab, tube, and bubble [13, 14]. These exotic nuclei are often called "nuclear pasta." Not only the density region of the pasta phases but also the charge number of roughly spherical nuclei is known to be sensitive to the empirically uncertain density dependence of the symmetry energy [15, 16]. Generally, it is difficult to observationally probe the properties of matter in the crust, but the asteroseismology could exceptionally help to constrain the matter properties such as nuclei present and the equation of state (EOS) (e.g., Refs. [17-20]). In this Letter, we will show by systematic analyses that an approach to the QPOs in SGR giant flares in terms of the torsional shear modes in the crust could severely constrain the density dependence of the symmetry energy.

We begin with the bulk energy per nucleon near the saturation point of symmetric nuclear matter at zero temperature, which can be written as a function of nucleon density $n$ and neutron excess $\alpha$ 21]:

$$
w=w_{0}+\frac{K_{0}}{18 n_{0}^{2}}\left(n-n_{0}\right)^{2}+\left[S_{0}+\frac{L}{3 n_{0}}\left(n-n_{0}\right)\right] \alpha^{2},
$$

where $w_{0}, n_{0}$, and $K_{0}$ are the saturation energy, saturation density, and incompressibility of symmetric nuclear matter, respectively. The parameters $L$ and $S_{0}$ characterize the symmetry energy coefficient $S(n): S_{0}=S\left(n_{0}\right)$ is the symmetry energy coefficient at $n=n_{0}$, while $L=3 n_{0}(d S / d n)_{n=n_{0}}$ is the symmetry energy density derivative coefficient. The parameters $w_{0}, n_{0}$, and $S_{0}$ can be relatively easier to determine from empirical masses and radii of stable nuclei 22]. On the other hand, the remaining two parameters, $L$ and $K_{0}$, are more difficult to determine. Here we introduce a new parameter, $y$, defined as $y=-K_{0} S_{0} /\left(3 n_{0} L\right)$, which denotes the slope of the saturation line in the vicinity of $\alpha=0$ 22]. Two of us (K.O. and K.I.) constructed the model for the EOS of nuclear matter in such a way as to reproduce Eq. (1) in the limit of $n \rightarrow n_{0}$ and $\alpha \rightarrow 0$, calculated the optimal density distribution of stable nuclei within a simplified version of the extended Thomas-Fermi theory, and obtained the values of $w_{0}, n_{0}$, and $S_{0}$ for given $y$ (or $L$ ) 
TABLE I: The pasta density region calculated for several sets of the EOS parameters.

\begin{tabular}{ccccc}
\hline \hline$y\left(\mathrm{MeV} \mathrm{fm}^{3}\right)$ & $K_{0}(\mathrm{MeV})$ & $L(\mathrm{MeV})$ & $n_{1}\left(\mathrm{fm}^{-3}\right)$ & $n_{2}\left(\mathrm{fm}^{-3}\right)$ \\
\hline-220 & 180 & 52.2 & 0.060 & 0.079 \\
-220 & 230 & 73.4 & 0.064 & 0.073 \\
-220 & 360 & 146.1 & 0.066 & 0.066 \\
-350 & 180 & 31.0 & 0.058 & 0.091 \\
-350 & 230 & 42.6 & 0.063 & 0.086 \\
-350 & 360 & 76.4 & 0.072 & 0.076 \\
-1800 & 180 & 5.7 & 0.058 & 0.134 \\
-1800 & 230 & 7.6 & 0.058 & 0.127 \\
-1800 & 360 & 12.8 & 0.058 & 0.118 \\
\hline \hline
\end{tabular}

and $K_{0}$ by fitting the charge number, mass excess, and charge radius that can be calculated from the optimal density distribution to the empirical behavior. In a manner similar to Ref. [15], we adopt the parameter range satisfying $0<L<160 \mathrm{MeV}, 180 \mathrm{MeV} \leq K_{0} \leq 360$ $\mathrm{MeV}$, and $y<-200 \mathrm{MeV} \mathrm{fm}^{3}$. In fact, this parameter range equally well reproduces the mass and radius data for stable nuclei and effectively covers even extreme cases [22]. With such parameters, we can obtain the crust EOS and the equilibrium nuclear shape and size by generalizing the Thomas-Fermi model for nuclei to matter in the crust. The results for the nucleon densities $n_{1}$ at which the nuclear shape changes from spherical to cylindrical one and $n_{2}$ at which the nuclear matter becomes uniform were tabulated for several sets of $y$ (or $L$ ) and $K_{0}$ in Table I. Note that the interval between $n_{1}$ and $n_{2}$, which corresponds to the pasta region, decreases with $L$ and vanishes at $L \sim 100 \mathrm{MeV}$.

We turn to the equilibrium structure of nonrotating neutron stars, which is determined by the TolmanOppenheimer-Volkoff (TOV) equations for given EOS of neutron star matter. For a solution to these equations, the metric can be described in terms of the spherical polar coordinates $r, \theta$, and $\phi$ as

$$
d s^{2}=-\mathrm{e}^{2 \Phi(r)} d t^{2}+\mathrm{e}^{2 \Lambda(r)} d r^{2}+r^{2} d \theta^{2}+r^{2} \sin ^{2} \theta d \phi^{2} .
$$

However, the EOS for the core surrounded by the crust is still uncertain, although there are many calculations based on realistic nuclear interactions and even constraints from the observationally deduced neutron star masses and radii [23, 24]. To avoid this uncertainty, we here construct the crust with the EOS models mentioned above by solving the TOV equations inward from the surface of the star for given stellar mass $M$ and radius $R$ as in Ref. [25]. We remark that the thickness of the crust thus constructed is consistent with a typical behavior given in terms of $M$ and $R$ by Eq. (18) in Ref. [26].

We next consider the shear modulus of a crustal part composed of spherical nuclei of charge $Z e$ and number density $n_{i}$, which can be approximately described as [27]

$$
\mu=0.1194 n_{i}(Z e)^{2} / a,
$$

where $a=\left(3 / 4 \pi n_{i}\right)^{1 / 3}$ is the Wigner-Seitz radius. Note that this formula is derived in the limit of zero temperature from Monte Carlo calculations of the shear modulus averaged over all directions for a perfect bcc Coulomb crystal of point charges embedded in a neutralizing uniform background [28]. We will use this formula for calculations of the torsional oscillation frequencies since effects of quantum zero-point motions and thermal fluctuations are negligible. For pasta nuclei except bubbles, the elasticity is expected to be much lower than that for spherical nuclei [29]. Moreover, the core, which is expected to be composed mostly of fluids, may have a structure with nonvanishing elasticity 30]. Here, we simply assume $\mu=0$ at $n>n_{1}$, as in Ref. [12]. Within this assumption, the shear modulus at $n>n_{1}$ is underestimated, which tends to lower the torsional oscillation frequencies. The frequencies as will be estimated below should thus be regarded as lower limits basically, but still will be shown to play a role in constraining $L$.

Since the torsional oscillations on a spherically symmetric star are incompressible, the star is free from deformation and density variation during such oscillations. One can thus determine the frequencies of the torsional oscillations with satisfactory accuracy even if one neglects the resulting metric perturbations by setting $\delta g_{\mu \nu}=0$, which is known as the relativistic Cowling approximation. Within this approximation, the torsional oscillations can be described by a single perturbation variable, i.e., the angular displacement of the stellar matter, $\mathcal{Y}$, which is related to the $\phi$-component of the perturbed 4-velocity of a matter element, $\delta u^{\phi}$, by $\delta u^{\phi}=\mathrm{e}^{-\Phi} \partial_{t} \mathcal{Y}(t, r) \partial_{\theta} P_{\ell}(\cos \theta) / \sin \theta$ with the $\ell$-th order Legendre polynomial $P_{\ell}$. Assuming $\mathcal{Y}(t, r)=\mathrm{e}^{\mathrm{i} \omega t} \mathcal{Y}(r)$, one can obtain the perturbation equation for $\mathcal{Y}(r)$ from the linearized equation of motion as 31.

$$
\begin{aligned}
\mathcal{Y}^{\prime \prime} & +\left[\left(\frac{4}{r}+\Phi^{\prime}-\Lambda^{\prime}\right)+\frac{\mu^{\prime}}{\mu}\right] \mathcal{Y}^{\prime} \\
& +\left[\frac{\epsilon+p}{\mu} \omega^{2} \mathrm{e}^{-2 \Phi}-\frac{(\ell+2)(\ell-1)}{r^{2}}\right] \mathrm{e}^{2 \Lambda} \mathcal{Y}=0
\end{aligned}
$$

where $\epsilon$ and $p$ are the energy density and pressure, and the prime denotes the derivative with respect to $r$. To determine the eigenfrequencies, we adopt the zero-traction condition at $n=n_{1}$ and the zero-torque condition at the star's surface. These boundary conditions reduce to $\mathcal{Y}^{\prime}=0$ at $n=0, n_{1}[5,31$. We remark that neutron superfluidity ignored here would subtract the mass density of superfluid neutrons from the enthalpy density $\epsilon+p$ and hence enhance the eigenfrequencies 32 .

First, to see the dependence of the fundamental torsional oscillations on the EOS parameters, we calculate 


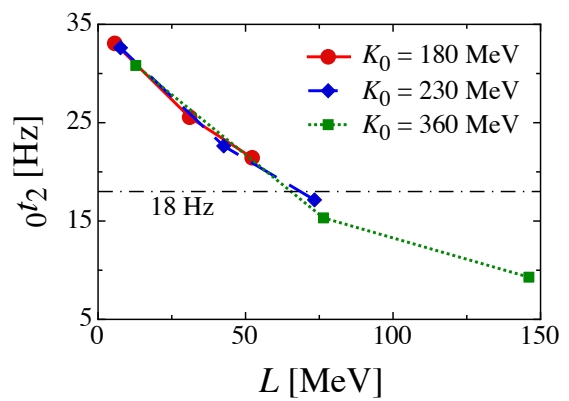

FIG. 1: (Color online) Frequency of the $\ell=2$ fundamental torsional oscillations, ot $t_{2}$, plotted as a function of $L$ for $M=1.4 M_{\odot}$ and $R=12 \mathrm{~km}$. The horizontal dot-dashed line denotes the lowest QPO frequency observed from SGR 1806-20 [1].

TABLE II: $\chi^{2}$ fitting via formula (5) for $M=1.4 M_{\odot}$, where $\chi^{2}$ is given for the deviation of the calculated ${ }_{0} t_{2}$ from the fitting formula (5).

\begin{tabular}{ccccc}
\hline \hline$R(\mathrm{~km})$ & $c_{0}(\mathrm{~Hz})$ & $c_{1}(\mathrm{~Hz})$ & $c_{2}(\mathrm{~Hz})$ & $\chi^{2}$ \\
\hline 10 & 38.95 & 36.14 & 11.34 & 1.944 \\
12 & 34.87 & 32.33 & 10.14 & 1.539 \\
14 & 31.48 & 29.14 & 9.123 & 1.239 \\
\hline \hline
\end{tabular}

the corresponding eigenfrequency for a typical stellar model with $M=1.4 M_{\odot}$ and $R=12 \mathrm{~km}$. Figure 1 shows the frequency of the $\ell=2$ fundamental torsional oscillations, ${ }_{0} t_{2}$, calculated for the nine sets of $L$ and $K_{0}$ that are tabulated in Table I together with the lowest QPO frequency in SGR 1806-20 [1]. From this figure, one can observe that ${ }_{0} t_{2}$ is almost independent of $K_{0}$ in the parameter range adopted here once the stellar model is fixed. Such independence can be seen for various stellar models ranging $R=10,12$, and $14 \mathrm{~km}$ as well as $M=1.4 M_{\odot}$ and $1.8 M_{\odot}$. We can thus focus on the $L$ dependence of the calculated ${ }_{0} t_{2}$, which arises mainly because the nuclear charge $Z$ decreases with $L$ through the surface property [15], leading to decrease in the shear modulus (3) with $L$. Since $Z$ depends strongly on $L$ in the vicinity of $n=n_{1}$, this region is likely to play a role in constraining $L$ via the evaluations of ${ }_{0} t_{2}$. To see the $L$ dependence explicitly, we derive a fitting formula for ${ }_{0} t_{2}$ as

$$
{ }_{0} t_{2}=c_{0}-c_{1} \frac{L}{100 \mathrm{MeV}}+c_{2}\left(\frac{L}{100 \mathrm{MeV}}\right)^{2},
$$

where $c_{0}, c_{1}$, and $c_{2}$ are the adjustable parameters that depend on $M$ and $R$. The values of these parameters for $M=1.4 M_{\odot}$ are shown in Table

The fitting formula thus obtained is exhibited in Fig. 2. We find from this figure that ${ }_{0} t_{2}$ for fixed $L$ can be determined within the accuracy of $\sim 20 \%$, if $R$ is in the

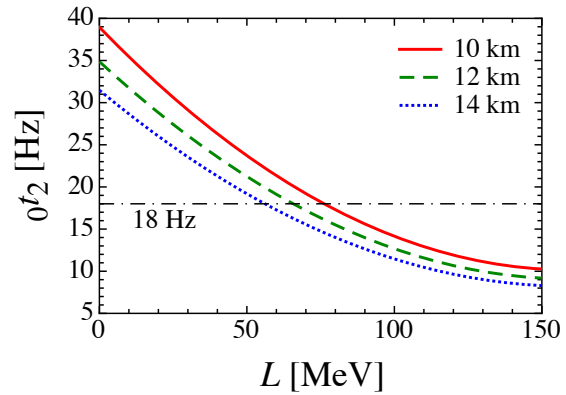

FIG. 2: (Color online) ${ }_{0} t_{2}$ given by formula (5) for $M=$ $1.4 M_{\odot}$ and $R=10,12,14 \mathrm{~km}$.

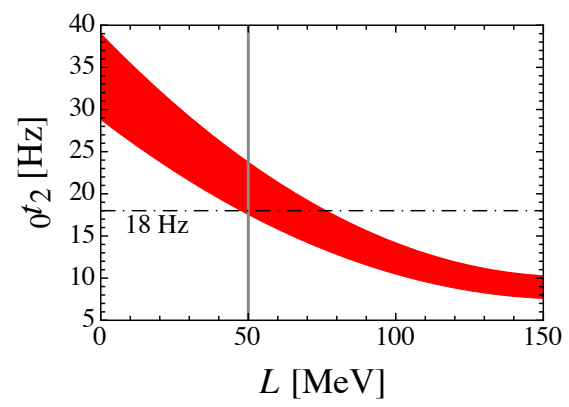

FIG. 3: (Color online) Same as Fig. 2 for $10 \mathrm{~km} \leq R \leq 14$ $\mathrm{km}$ and $1.4 M_{\odot} \leq M \leq 1.8 M_{\odot}$.

range of $10-14 \mathrm{~km}$. By shifting $M$ from $1.4 M_{\odot}$ up to $1.8 M_{\odot}$, we find that ${ }_{0} t_{2}$ for $R=10,12$, and $14 \mathrm{~km}$ decreases only by $\sim 14,10$, and $9 \%$, respectively. For clarity, we plot, in Fig. 3. ot $t_{2}$ estimated for stellar models ranging $10 \mathrm{~km} \leq R \leq 14 \mathrm{~km}$ and $1.4 M_{\odot} \leq M \leq 1.8 M_{\odot}$. The results are confined within the painted region, which is so narrow that we can constrain $L$ as we shall see.

Let us assume that the observed QPOs in SGR giant flares arise from the torsional oscillations in neutron star crusts and note that among many eigenfrequencies of the torsional oscillations, ${ }_{0} t_{2}$ is the lowest. Then, ${ }_{0} t_{2}$ would become equal to or even lower than the lowest frequency in the observed QPOs. Consequently, one can constrain $L$ as $L \gtrsim 50 \mathrm{MeV}$ from the painted region in Fig. 3. Recall that the calculated ${ }_{0} t_{2}$ is likely to be underestimated because of the simplified treatments of the shear modulus and the enthalpy density. In particular, elasticity in the pasta phases ignored here would act to increase ${ }_{0} t_{2}$ as long as $n_{2}>n_{1}$ [12]. At $L \gtrsim 50 \mathrm{MeV}$, however, $n_{2}-n_{1}$ is already small (see Table \). The resultant modifications on the $L$ constraint are thus expected to be small.

We proceed to show the frequency of the first overtone of the $\ell=2$ torsional oscillations, ${ }_{1} t_{2}$, calculated again for the nine sets of $L$ and $K_{0}$ that are tabulated in Table (1) The results with $M=1.4 M_{\odot}$ and $R=10,12,14 \mathrm{~km}$ are plotted in Fig. 4. We find from this figure that ${ }_{1} t_{2}$ 


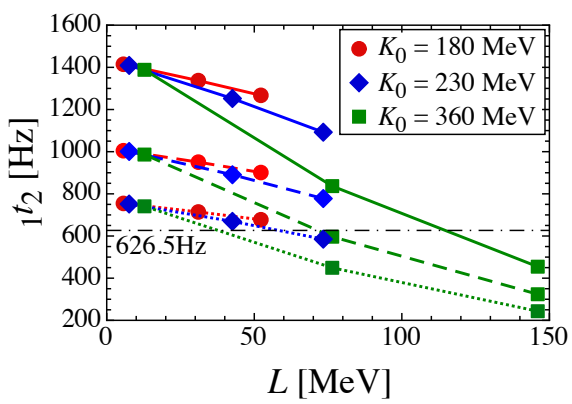

FIG. 4: (Color online) Frequency of the first overtone of the $\ell=2$ torsional oscillations, ${ }_{1} t_{2}$, plotted as a function of $L$ for $M=1.4 M_{\odot}$. The solid, broken, and dotted lines are the results for $R=10,12$, and $14 \mathrm{~km}$, while the circles, diamonds, and squares are those for $K_{0}=180,230$, and 360 $\mathrm{MeV}$. The horizontal dot-dashed line denotes one of the high QPO frequencies observed from SGR 1806-20 [1].

depends not only on $L$, but on $K_{0}$ and $R$ significantly, in contrast to the case of ${ }_{0} t_{2}$. The $R$ dependence arises because basically, ${ }_{1} t_{2}$ is inversely proportional to the crust thickness, which in turn increases as $R^{2}$ [26]. The $K_{0}$ dependence comes presumably from the $K_{0}$ dependence of $n_{1}$ (see Table 【), but could be modified drastically once elasticity in the pasta phases is allowed for. This issue will be addressed elsewhere 33.

In summary, we have investigated the fundamental torsional mode and the first overtone in neutron star crusts for various EOS and stellar models. The identification of the lowest QPO frequency observed from SGR 1806-20 as ${ }_{0} t_{2}$ would then allow us to constrain $L$ as $L \gtrsim 50 \mathrm{MeV}$. At present, this constraint is fairly stringent because experimental constraints on $L$ have yet to converge 34]. While there are earlier publications that remark the sensitivity of ${ }_{0} t_{2}$ and ${ }_{1} t_{2}$ to $L[10,12$, the present work is the first to provide a lower limit of $L$ by sufficiently accurate and systematic calculations through the general relativistic mode evaluations and the Thomas-Fermi treatment of nuclei. The present constraint already suggests that the pasta phases, if any in neutron stars, would occur in a narrow density region. Neutron superfluidity and pasta elasticity would make the constraint on $L$ and the pasta region even more stringent.

H.S. is grateful to T. Tatsumi for fruitful discussions. This work was supported in part by Grants-in-Aid for Scientific Research on Innovative Areas through No. 23105711 and on Research Activity Start-up through No. 23840038, which were provided by the Ministry of Education, Culture, Sports, Science, and Technology of Japan.

* Electronic address: hajime.sotani@nao.ac.jp
[1] A. L. Watts and T. E. Strohmayer, Adv. Space Res. 40, 1446 (2006).

[2] Y. Levin, Mon. Not. R. Astron. Soc. 368, L35 (2006).

[3] U. Lee, Mon. Not. R. Astron. Soc. 374, 1015 (2007).

[4] L. Samuelsson and N. Andersson, Mon. Not. R. Astron. Soc. 374, 256 (2007).

[5] H. Sotani, K. D. Kokkotas, and N. Stergioulas, Mon. Not. R. Astron. Soc. 375, 261(2007); 385, L5 (2008).

[6] H. Sotani, A. Colaiuda, and K. D. Kokkotas, Mon. Not. R. Astron. Soc. 385, 2161 (2008).

[7] H. Sotani and K. D. Kokkotas, Mon. Not. R. Astron. Soc. 395, 1163 (2009).

[8] M. Gabler et al., Mon. Not. R. Astron. Soc. 410, L37 (2011).

[9] A. Colaiuda and K. D. Kokkotas, Mon. Not. R. Astron. Soc. 414, 3014 (2011).

[10] A. W. Steiner and A. L. Watts, Phys. Rev. Lett. 103, 181101 (2009).

[11] H. Sotani, Mon. Not. R. Astron. Soc. 417, L70 (2011).

[12] M. Gearheart, W. G. Newton, J. Hooker, and B. A. Li, arXiv: 1106.4875

[13] C. P. Lorenz, D. G. Ravenhall, and C. J. Pethick, Phys. Rev. Lett. 70, 379 (1993).

[14] K. Oyamatsu, Nucl. Phys. A 561, 431 (1993).

[15] K. Oyamatsu and K. Iida, Phys. Rev. C 75, 015801 (2007).

[16] W. G. Newton, M. Gearheart, and B. A. Li, arXiv:1110.4043

[17] N. Andersson and K. D. Kokkotas, Phys. Rev. Lett. 677, 4134 (1996).

[18] H. Sotani, K. Tominaga, and K. I. Maeda, Phys. Rev. D 65, 024010 (2001).

[19] H. Sotani, K. Kohri, and T. Harada, Phys. Rev. D 69, 084008 (2004).

[20] H. Sotani, N. Yasutake, T. Maruyama, and T. Tatsumi, Phys. Rev. D 83, 024014 (2011).

[21] J. M. Lattimer, Annu. Rev. Nucl. Part. Sci. 31, 337 (1981).

[22] K. Oyamatsu and K. Iida, Prog. Theor. Phys. 109, 631 (2003).

[23] F. Özel, G. Baym, and T. Güver, Phys. Rev. D 82, 101301 (2010).

[24] A. W. Steiner, J. M. Lattimer, and E. F. Brown, Astrophys. J. 722, 33 (2010).

[25] K. Iida and K. Sato, Astrophys. J. 477, 294 (1997).

[26] D. G. Ravenhall and C. J. Pethick, Astrophys. J. 424, 846 (1994).

[27] T. Strohmayer et al., Astrophys. J. 375, 679 (1991).

[28] S. Ogata and S. Ichimaru, Phys. Rev. A 42, 4867 (1990).

[29] C. J. Pethick and A. Y. Potekhin, Phys. Lett. B 427, 7 (1998).

[30] N. K. Johnson-McDaniel and B. J. Owen, arXiv:1110.4650

[31] B. L. Schumaker and K. S. Thorne, Mon. Not. R. Astron. Soc. 203, 457 (1983).

[32] C. J. Pethick, N. Chamel, and S. Reddy, Prog. Theor. Phys. Suppl. 186, 9 (2010).

[33] H. Sotani, K. Nakazato, K. Iida, and K. Oyamatsu (unpublished).

[34] See, e.g., B. A. Li, C. W. Chen, and C. M. Ko, Phys. Rep. 464, 113 (2008); M. B. Tsang et al., Phys. Rev. Lett. 102, 122701 (2009). 